\title{
ANÁLISE TÉCNICA DA VIABILIDADE ECONÔMICA DO CULTIVO, EM LARGA ESCALA, DA TERMINALIA CATAPPA LINN, NO RENDIMENTO DE ÓLEO PARA FINS ENERGÉTICOS.
}

\author{
L. D. SILVA NETO ${ }^{1}$, E. N. SILVA ${ }^{1}$, C.M.B. PEREIRA ${ }^{2}$, J. I. SOLETTI ${ }^{1}$ e S.H.V. CARVALHO ${ }^{1}$. \\ ${ }^{1}$ Universidade Federal de Alagoas, Departamento de Engenharia Química \\ ${ }^{2}$ Universidade Federal de Alagoas, Departamento de Ciências Agrárias \\ E-mail para contato:1d_neto@hotmail.com
}

\begin{abstract}
RESUMO - Um dos grandes desafios do século XXI é desenvolver matrizes energéticas "limpas" a partir de fontes renováveis de baixo custo, e que preserve o meio ambiente. Uma das energias que pode atender a essa necessidade é a proveniente da biomassa, a exemplo dos óleos vegetais na produção do biodiesel. Neste trabalho avaliamos a caracterização do óleo da amêndoa do fruto da Terminalia Catappa Linn - TC, visando seu uso na produção do biodiesel, a partir de frutos secos. Além disso, foi feita, a análise da viabilidade econômica do cultivo dessa espécie em larga escala, visando o rendimento de óleo para fins energéticos, em comparação com outras oleaginosas brasileiras. Os resultados se enquadram nos padrões mínimos de qualidade exigidos pela ANVISA/1999 e a analise da viabilidade econômica do cultivo, em larga escala, no rendimento de óleo para fins energéticos, demonstrou que a perspectiva de rendimento por produção de óleo foi aproximadamente de 0,6 t. (t de óleo/ha ano).
\end{abstract}

\section{INTRODUÇÃO (FONTE 14)}

A matriz elétrica brasileira tem uma participação de $88,8 \%$ de fontes renováveis. A média mundial é $19,5 \%$ e, entre os países membros da Organização para a Cooperação e Desenvolvimento Econômico (OCDE), a média é 18,3\%. (MME, 2015).

Qualquer matéria orgânica que possa ser transformada em energia mecânica, térmica ou elétrica é classificada como biomassa. O uso planejado de forma sustentável da biomassa traz muitos benefícios ambientais e sociais, se comparada com os combustíveis fósseis, pois além do uso de terras agrícolas excedentes em países em desenvolvimento, utiliza os resíduos promovendo a reciclagem da matéria e gerando emprego (Rosillo, et al., 2005). Além disso, a biomassa possui um ciclo extremamente curto e maiores benefícios ambientais e energéticos podem derivar do cultivo de plantas perenes e florestas, além de plantações com safras anuais, que são matéria-prima alternativa de curto prazo para a produção de combustíveis (Hall, et al., 2005).

\subsection{Caracterização da Amendoeira (Terminalia Catappa Linn)}

As espécies do gênero Terminalia, pertencem à família Combretácea que é formada por cerca de 600 espécies e entre os seus gêneros, destacasse dois de maior ocorrência, o 
Combretum e o Terminalia, ambos contendo 250 espécies (Francis, et al.,1989). A Terminalia Catappa Linn é uma árvore exótica, que cresce em regiões tropicais e subtropicais, localizadas em áreas costeiras. Esta arvore foi introduzida em muitos países tropicais do mundo, a exemplo do Brasil, devido à migração humana. A árvore tem de 6-12 metros de altura, podendo chegar até 20 metros.

Os frutos são drupas elipsoides biangulados, de $3-5 \mathrm{~cm}$ de comprimento, de cor, que varia entre o roxo e o amarelo quando maduros, com polpa carnosa, contendo em seu interior uma semente ovalada e rica em óleo, envolvida por uma casca muito dura (Instituto Plantarum, 2005). A produção de amêndoas é estimada em cerca de $5 \mathrm{~kg}$ por árvore ao ano, pode ser o dobro a partir de estirpes geneticamente selecionadas e cultivadas em lugares de alta qualidade. As amêndoas são comestíveis, mas a sua qualidade e tamanho são variáveis.

\section{METODOLOGIA}

As amostras de amêndoas foram coletadas de árvores distintas, período de novembro de 2015 a março de 2016 na cidade de Maceió. Uma parte foi de amendoeira do Campus da Universidade, e o restante foi coletado nas intermediações da cidade. Estes foram postos para secagem, ao ar livre, durante 15 dias. Após a retirada das amêndoas, estas foram desidratadas em estufa por 24 horas a uma temperatura em torno de $60^{\circ} \mathrm{C}$. O óleo foi extraído em uma prensa hidráulica da marca Tecnal.

A caracterização deste produto é de extrema importância uma vez que a composição deste óleo pode ser decisiva na definição de seus usos potenciais, pois, na obtenção e emprego dos óleos vegetais, tanto para fins alimentícios como o emprego desses óleos diretamente na formulação de combustíveis minerais, o conhecimento de suas propriedades físicas e químicas é de fundamental importância para seu uso. O teor de umidade, massa especifica, viscosidade cinemática, índice de acidez, índice de saponificação e o pH, além do teor de lipídios e a composição dos ácidos graxos foram feitas seguindo normas oficiais utilizadas, comumente em cada uma delas. Para a determinação do teor de lipídios, o teor de óleo presente na amostra da amêndoa foi determinado através da extração por solvente, utilizando o método Soxhlet padrão, com hexano como solvente, onde utilizasse aproximadamente $10 \mathrm{~g}$ da amostra da amêndoa, in natura.

\subsection{Determinações da composição dos Ácidos graxos no óleo da amêndoa da Terminalia catappa linn.}

A identificação dos ácidos graxos da amêndoa foi determinada por cromatografia em fase gasosa (CG), sendo empregado um cromatógrafo da Shimadzu, modelo ZBWAXplus detector de ionização de chama (FID) operando a $250^{\circ} \mathrm{C}$. A análise foi realizada através de uma adaptação do método prescrito pela norma europeia EN 14103, onde a amostra foi dissolvendo, aproximadamente, $0,0500 \mathrm{~g}$ em da amostra em 1,0 ml de hexano. Em seguida os compostos foram separados em coluna capilar apolar ZB-WAXplus com $30.0 \mathrm{~m}$ de comprimento, $0,32 \mathrm{~mm}$ de diâmetro interno e filme com $0,25 \mu \mathrm{m}$ de espessura e gás hidrogênio, de alta pureza (99,95\% LINDE), Em seguida foi empregada uma coluna capilar apolar ZB-WAXplus com $30.0 \mathrm{~m}$ de comprimento, $0,32 \mathrm{~mm}$ de diâmetro interno e filme com $0,25 \mu \mathrm{m}$ de espessura e gás hidrogênio, de alta pureza (99,95 \% LINDE), usado como gás de arraste. A programação de temperatura foi: temperatura constante de $200^{\circ} \mathrm{C}(10 \mathrm{~min})$; A 
composição em ácidos graxos foi calculada com base na identificação e integração das áreas dos picos por normalização.

\section{RESULTADOS E DISCUSSÕES}

Os resultados das análises físico-químicas do óleo da amendoeira (Terminalia catappa linn) em seu estado in natura, comparado com valores encontrados em literatura encontramse apresentados na Tabela 1. Vale ressaltar, que o óleo utilizado para caracterização dos parâmetros físico-químicos, foi extraído por prensagem mecânica a frio.

Tabela 1: Resultados dos parâmetros analíticos realizados com o óleo da Terminalia catappa linn - TC comparado aos resultados encontrados para a soja.

\begin{tabular}{|c|c|c|c|}
\hline \multicolumn{4}{|c|}{ Caracterização do óleo da Terminalia catappa Linn (TC) } \\
\hline Parâmetros analíticos realizados & $\begin{array}{c}\text { TC } \\
\text { Resultados } \\
\text { experimentais }\end{array}$ & $\begin{array}{c}\text { TC } \\
\text { SOUZA, et } \\
\text { al,2015 }\end{array}$ & $\begin{array}{c}\text { Óleo de soja } \\
\text { ALMEIDA, } \\
\text { k.P.A., et al, } 2011\end{array}$ \\
\hline Umidade $(\%)$ & 0,73 & nd & nd \\
\hline Massa específica $\left(\mathrm{kg} / \mathrm{ml}\right.$ a $\left.20^{\circ} \mathrm{c}\right)$ & 0,916 & 0,905 & 0,945 \\
\hline Viscosidade $\left(\mathrm{mm}^{2} \mathrm{~s}^{-1} \mathrm{a} 40^{\circ} \mathrm{c}\right)$ & 42,00 & 38,05 & 92,3 \\
\hline Índice de acidez (\%AGL) & 0,26 & 3,35 & 0,61 \\
\hline Índice de saponificação (mg NaOH,) & 285,0 & 173,91 & 142,32 \\
\hline $\mathrm{pH}$ & 6,00 & nd & --- \\
\hline Teor de lipídios na amêndoa ( \%) & 56,00 & 53,00 & -- \\
\hline
\end{tabular}

Fonte: Autor (2017)

Pode-se observar, através da Tabela 6, que os resultados encontrados são aproximados aos resultados encontrados por Souza et al., (2015). Algumas das propriedades analisadas divergem das leis para produção de biodiesel especificada na ANP/2012. Porem, os resultados se enquadram nos padrões mínimos de qualidade exigidos pela ANVISA/1999.

Com relação ao teor de lipídios, os resultados demonstram que a amendoeira (Terminalia catappa linn), apresentou um alto rendimento em óleo (56\%), resultado aproximado do resultado encontrado por Souza et. al., (2015), (53\%). Fazendo uma comparação com outras oleaginosas brasileiras, que segundo Silva (2009), apresentam teores em óleo nas amostras como algodão (20-30\%), amendoim (45-50\%), gergelim (40-50\%), mamona (45-55\%) e soja (18-20\%), esses resultados, comprovam que a amendoeira tem uma quantidade considerável de óleo em sua amêndoa. Resultados que reforçam a viabilidade econômica em se trabalhar com o óleo da amendoeira, pois, segundo Brooker et al.,(1992), entre as propriedades que determinam a boa qualidade dos grãos, está o alto teor de óleo.

\subsection{Determinação da composição de Ácidos graxos presentes no óleo da amêndoa da Terminalia catappa lin.(TC)}

Foram identificados e quantificados os ácidos. O resultado foi exposto na Tabela 2, em comparação com os resultados encontrados por Souza et. al. (2015), para a mesma espécie em estudo. De acordo com a EMBRAPA (2008), quanto menor o número de insaturações (duplas ligações) nas moléculas, maior o número de cetano do combustível, ocasionando uma 
melhor "qualidade à combustão". Ainda segundo a EMBRAPA (2008), de uma forma geral, um biodiesel com predominância de ácidos graxos combinados monoinsaturados (oleico, ricinoléico) são os que apresentam os melhores resultados.

Tabela 2- Resultados da composição de ácidos graxos do óleo da Terminalia catappa linn comparado com os resultados encontrados por Souza et al.,(2015).

\begin{tabular}{ccc}
\hline & Resultados experimentais & SOUZA, et al.,(2015). \\
\hline AG & \%massa & \% massa \\
\hline C20:0 araquidônico & $\mathbf{0 , 5}$ & 0,3 \\
C14: 0 Mirístico & $\mathbf{0 , 1}$ & 0,07 \\
C16:0 palmítico & $\mathbf{3 7 , 6}$ & 34,2 \\
C18:0 esteárico & $\mathbf{2 , 7}$ & 4,2 \\
C18:1 oleico & $\mathbf{3 2 , 4}$ & 33,8 \\
C16:1 palmitoléico & $\mathbf{0 , 4}$ & -- \\
C18:2 linoleico & $\mathbf{2 6 , 4}$ & 22,2 \\
C18:3 linolênico & $\mathbf{0 , 1}$ & 0,06 \\
\hline
\end{tabular}

Fonte: Autor (2017)

Além da linha de biocombustível, a amendoeira vem sendo estudada, também para proposito nutricional. O óleo da amêndoa por se mostra rico em ácidos graxos insaturados, seu consumo pode apresentar diversos efeitos benéficos ao ser humano, desde prevenção até tratamento de doenças cardiovasculares, diabetes, câncer, processos inflamatórios, entre outros, agindo como uma fonte de alimentação funciona.

3.2 Analise técnica da viabilidade econômica do cultivo, em larga escala, da Terminalia catappa linn, no rendimento de óleo para fins energéticos.

Com base na Figura 1 é possível se estabeler a relação entre a área cultivada/área ocupada por uma planta, determinando assim a densidade de plantas por hectares, com isso tem-se, atraves da Equação 1:

$$
D=\frac{\mathrm{AC}}{\mathrm{A}}
$$

Onde: $\mathrm{D}=$ Densidade de plantas por $\mathrm{ha}^{-1} ; \mathrm{AC}=$ Área cultivada, $\mathrm{m}^{2} ; \mathrm{A}=$ Área de ocupação de uma planta, $\mathrm{m}^{2}$;

Com isso estima-se que em um hectare seja possível à implantação de aproximadamente 204 plantas e baseado em coeficientes técnicos de produção de óleo por planta, estima-se a produtividade de óleo/planta/hectare. Resultados convertido em toneladas, exposto na Tabela 3.

tabela 3 - cálculo da produção estimada de óleo por unidade agrícola (kg de óleo/ha/ano) e (t de óleo/ha/ano) da amendoeira (terminalia catappa l.).

\begin{tabular}{cccccc}
\hline $\begin{array}{c}\text { Quant. de } \\
\text { planta/ ha }\end{array}$ & $\begin{array}{c}\text { Quant.de } \\
\text { semente/planta/ } \\
\text { ano/kg }\end{array}$ & $\begin{array}{c}\text { (Quant. De } \\
\text { semente/ha/ano } \\
/ \mathbf{k g}\end{array}$ & $\begin{array}{c}\text { Teor de } \\
\text { óleo } \\
(\%)\end{array}$ & $\begin{array}{c}\text { (Kg/óleo/ } \\
\text { Ano/ha) }\end{array}$ & $\begin{array}{c}\text { Rendimento } \\
\text { t/óleo/ ha/ano }\end{array}$ \\
\hline 204 & 5 & 1020 & 60 & 612 & 0,6 \\
\hline
\end{tabular}


O resultado demonstrou que a perspectiva de rendimento em produção de óleo por unidade agrícola ( $\mathrm{t}$ de óleo/ha ano), foi aproximadamente de 0,6 toneladas. E comparado aos rendimentos de outras oleaginosas brasileiras, resultados encontrados por (Mourad, 2006), essa estimativa demostra que o cultivo em larga escala da Terminalia catappa $l$. no rendimento de óleo para fins energéticos, é viável economicamente. Resultados exposta na Tabela 4.

Figura 1- Área de ocupação de uma planta em campo

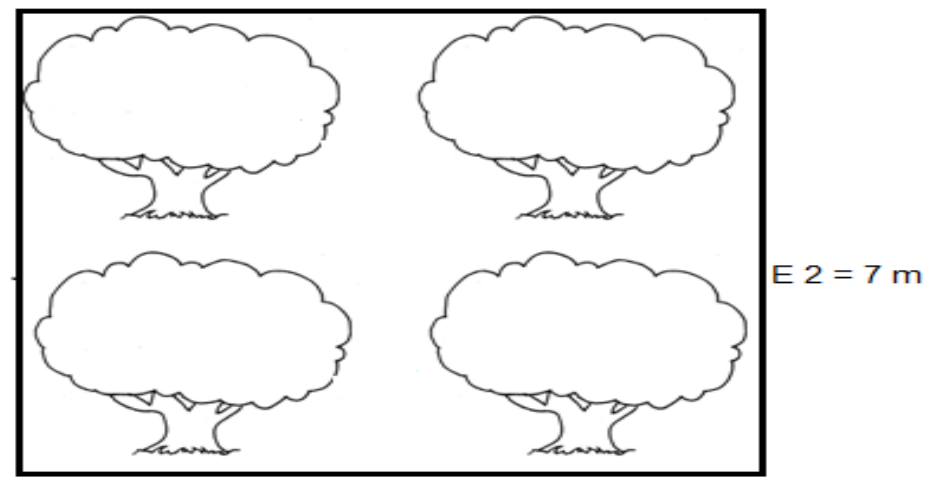

$$
\mathrm{E} 1=7 \mathrm{~m}
$$

$$
\begin{aligned}
& \text { Dados: } \\
& \text { E1 = Espaçamento entre linhas; } \\
& \text { E2 = Espaçamento entre } \\
& \text { planțas; } \\
& \text { A = Área de ocupação de uma } \\
& \text { planta }
\end{aligned}
$$

Fonte: Santos, 2016

$$
\begin{aligned}
& A=E 1 \times E 2 \\
& A=7 \times 7 \\
& A=49 \mathrm{~m}^{2}
\end{aligned}
$$

Tabela 4 - Estimativa da produtividade de óleo/planta/hectare da amendoeira (Terminalia catappa $l$.) em comparação com os resultados encontrados nas principais oleaginosas brasileiras.

\begin{tabular}{cccc}
\hline Espécie & Teor de óleo $(\%)$ & Meses de colheita/ano & Rendimento (t) óleo/ano \\
\hline Amendoeira & $\mathbf{6 0}$ & $\mathbf{5}$ & $\mathbf{0 , 6}$ \\
Dendê/ palma & 22 & 12 & $3-6$ \\
Coco & $55-60$ & 12 & $1,3-1,9$ \\
Babaçu & 66 & 12 & $0,1-0,3$ \\
Girassol & $38-48$ & 3 & $0,5-1,9$ \\
Colza / canola & $40-48$ & 3 & $0,5-0,9$ \\
Mamona & $45-50$ & 3 & $0,5-0,9$ \\
Amendoim & $40-43$ & 3 & $0,6-0,8$ \\
Soja & 18 & 3 & $0,2-0,4$ \\
Algodão & 15 & 3 & $0,1-0,2$ \\
\hline
\end{tabular}

Fonte: Adaptada de MAPA, 2005.

\section{CONCLUSÕES}

Comparando a perspectiva de rendimento de 0,6 t óleo/ha/ano, da Terminalia catappa $l$., com resultados encontrados na literatura para as principais oleaginosas brasileiras, a espécie está dentro da média. Estes resultados apresentam a amendoeira com um rendimento maior que o da soja, padrão brasileiro na produção de óleos, a qual apresenta um rendimento médio de $0,3 \mathrm{t}$ óleo/ha/ano, e próximo aos da canola, mamona e amendoim, ambos apresentam uma média de $0,7 \mathrm{t}$ óleo/ha/ano. Vale ressaltar, ainda, que essa estimativa de rendimento em 
óleo para a amendoeira (TC), foi baseada em um cultivo sem processo de adubação e irrigação, em áreas degradadas. Além disso, é possível compensar o tempo de frutificação e colheita (aproximadamente três anos após o plantio) com uma forma de cultivo consorciado com outras espécies não perene, como mandioca, feijão entre outras.

Com isso, a amendoeira (Terminalia Catappa L.), com sua perspectiva de um bom rendimento em óleo, comprova a viabilidade do seu cultivo, em larga escala, para complementar esse mercado de oleaginosa na produção de biodiesel.

\section{REFERÊNCIAS}

BROOKER, D.B.; BAAKER-ARKEMA,F.W.;HALL,C.W. Drying and Storage of Grain and Oilseeds . New York. USA. 1992. 450p.

EMBRAPA - EMPRESA BRASILEIRA DE PESQUISA AGROPECUÁRIA. Oleaginosas e seus óleos: Vantagens e Desvantagens para Produção de Biodiesel, por Napoleão Esberard de Macêdo Beltrão e Maria Isaura Pereira de Oliveira. Campina Grande, 2008. 28p. (Embrapa Algodão. Documentos, 201).

FRANCIS, 1989; LIN, 1992; THOMSON \& EVANS, 2006, apud TEIXEIRA, H.L. Composição Química E Perfil De Ácidos Graxos Da Castanha Do Fruto Da Castanhola (Terminalia Catappa Linn). 2010. 60p. (Dissertação - Mestrado) Programa de Pós - Graduação em Engenharia de Alimentos, Área Concentração em Engenharia de Processo. Universidade Estadual Do Sudoeste Da Bahia - UESB, Campus de Itapetinga-BA

HALL, D. O.; HOUSE, J. I.; SCRASE, I. Visão geral de energia e biomassa. In: rosillocalle, f.; bajay, s. v.; rothman, h. (Org.). Uso da biomassa para produção de energia na indústria brasileira. Campinas: UNICAMP, 2005. Cap. 1, p. 25-67.

INSTITUTO PLANTARUM. Amendoeira. 2005. Página disponível em: <http://www.plantarum.com.br/amendoeira.html>. Acesso em jul. 2016.

MAPA - MINISTÉRIO DA AGRICULTURA, PECUÁRIA E ABASTECIMENTO - Plano Nacional de Agroenergia, Brasília DF, 2005.

MME - MINISTÉRIO DE MINAS E ENERGIA. Balanço energético nacional 2015: ano base 2014. Brasília, DF 1982. 105p.

MOURAD, A.L. Principais Culturas Para Obtenção De Óleos Vegetais Combustíveis No Brasil, Encontro de Energia No Meio Rural, An. 06 /2006.

SILVA, I. C. C. Uso de processos combinados para o aumento do rendimento da extração e da qualidade do óleo de macaúba. Dissertação (Mestrado em Tecnologia de Processos Químicos e Bioquímicos), Universidade Federal do Rio de Janeiro, Rio de Janeiro, [s.n.], 2009.

SOUZA A.L.G., et al. Aproveitamento nutricional e tecnológico dos frutos da castanhola. Rev. Pan-Amazonica em Saúde 2015; 7(3):23-29p.

ROSILLO, F. BAJAY, S. ROTHAN, H. Uso da biomassa para produção de energia na indústria brasileira, $1^{\mathrm{a}}$ ed. São Paulo: editora da Unicamp, 2005.

SANTOS, J. R. T, Engenheiro Agrônomo Mestre em Produção Vegetal, responsável técnico, Engecampi Projetos e consultorias agroambientais, 2016. 